\title{
Fachliche Mängel im jüngsten Bericht des Swiss Medical Boards
}

\author{
Michel Romanens ${ }^{a}$, \\ Franz Ackermann ${ }^{b}$, \\ Flavian Kurthc \\ a Präsident Verein Ethik und \\ Medizin Schweiz (VEMS) \\ b Vorstand VEMS \\ c Sekretär VEMS
}

Eine Stellungnahme der FMH und des Swiss Medical Board findet sich im Anschluss auf den Seiten 1365 und 1367.

Korrespondenz:

Dr. med. Michel Romanens Facharzt für Innere Medizin und Kardiologie

Verein Ethik und Medizin

Schweiz (VEMS)

Ziegelfeldstrasse 1

CH-4600 Olten

info[at]kardiolab.ch

www.vems.ch

\section{Empfehlung des Swiss Medical Boards}

In seinem jüngsten Bericht «ComputertomographieScanner in der Abklärung der koronaren Herzerkrankung» empfiehlt das Swiss Medical Board (SMB), anstelle der invasiven Koronarangiographie (IKA) vermehrt die kardiale Computertomographie (KCT) mit Geräten der neueren Generation (64-Zeiler und mehr) einzusetzen. Davon erhofft sich das SMB eine Kostensenkung bei der Abklärung der vermuteten koronaren Herzkrankheit (KHK). Zusammenfassend empfiehlt das Medical Board, vor der Durchführung eines bildgebenden Verfahrens bei Patienten mit einer vermuteten KHK die Vortestwahrscheinlichkeit sorgfältig abzuschätzen, bei Vorliegen einer solchen von unter $10 \%$ auf das bildgebende Verfahren ganz zu verzichten, bei einer solchen von 10 bis 30\% das bildgebende Verfahren möglichst zu vermeiden und wenn, dann a priori kein IKA anzuwenden, sowie bei einer Vortestwahrscheinlichkeit von 30 bis 50\% als erstes bildgebendes Verfahren eine kardiale Computertomographie KCT durchzuführen, um bei positiven Befunden gegebenenfalls mit einer IKA weiter abzuklären und eventuell zu therapieren.

\section{Lacunes techniques dans le récent rapport du Swiss Medical Board}

Le présent article met en cause le rapport du Swiss Medical Board «Usage de la tomodensitométrie pour le diagnostic de la maladie coronarienne» dont le volet technique repose, selon les auteurs, sur des bases erronées. II pointe du doigt le manque de réflexion sur les incidences d'un tel rapport sur la pratique clinique, celles-ci étant en effet susceptibles de chambouler entièrement, et non sans danger, un chemin clinique bien établi en cardiologie, de nuire à l'activité des médecins et de conduire aussi bien à une augmentation des coûts qu'à la mise en danger des patients. La question se pose de savoir si les deux sociétés de discipline concernées, et nommément les deux experts que s'est adjoint le Swiss Medical Board, peuvent signer ce rapport en toute conscience. 


\section{Klinische Praxis}

Die klinische Praxis sieht anders aus, und dies mit gutem Grund: Im Rahmen der Abklärung von Brustbeschwerden werden in der Schweiz Personen mit auf koronare Herzkrankheit verdächtigen Brustbeschwerden mittels eines Belastungs-Elektrokardiogramms abgeklärt. Damit lassen sich für rund 250 CHF kostengünstig die meisten Koronarpatienten erfassen oder ausschliessen. Wegen der Sensitivität und Spezifität des Tests verbleiben jedoch einige Personen im intermediären Risikosegment für eine koronare Herzkrankheit. Bei diesen wird normalerweise ein Bildgebungstest mit funktioneller Belastung angeschlossen, beispielsweise eine BelastungsEchokardiographie zum Preis von rund 500 CHF. Dieser Test erlaubt ohne jegliche Strahlenbelastung eine Quantifizierung, nicht nur betreffend Diagnostik für das Vorliegen einer koronaren Herzkrankheit, sondern auch betreffend Prognose. Denn selbst bei Vorliegen einer nicht ausgedehnten Durchblutungsstörung des Herzens ist primär ein konservatives Vorgehen durchaus eine Option. Damit verbleiben praktisch keine Personen mehr im intermediären Risikosegment, sodass mit hoher Sicherheit auf ein Koronarangiogramm verzichtet werden kann. Dieser etablierte Abklärungspfad wird, allenfalls unter Einschluss von Herzinfarkt-Markern (Troponin-Test), auch im Spital bei wegen Brustbeschwerden hospitalisierten Personen angewendet. Der Abklärungsschritt für ein Koronarangiogramm mittels KCT oder IKA findet in der Regel im Spital nicht ohne Ischämietest und nicht bei normalem Troponin statt. Der direkte Gang zum KCT ist demnach nicht der wirksame Weg für eine optimale Abklärung und Betreuung der Patienten mit Brustbeschwerden.

\section{Falsche Beurteilungsgrundlagen}

Die Grundlagen für die anderslautenden Empfehlungen des SMB sind eindeutig mangelhaft erarbeitet. Zunächst ist zu bemängeln, dass das Medical Board in seiner Beurteilung die nicht invasive Ischämiediagnostik ausblendet, ohne die Gründe dafür offenzulegen. Alsdann folgen banale Rechnungsfehler: Die Kosten für ein KCT betragen nicht, wie im Bericht auf Seite 20 falsch berechnet, 467 CHF, sondern 867 CHF. Hier hat das Medical Board vergessen, die Kosten für das Kontrastmittel (170 CHF) und für den Ca-Score (219 CHF) mit einzurechnen.

Auch bei der Berechnung der Kosten für das IKA sind dem SMB Fehler unterlaufen: Bei einem Kostengewicht von 0,539 können aufgrund unterschiedlicher Baserates je nach Kanton und Spital verschiedene Kosten resultieren. Diese Unterschiede sind recht erheblich. Im Kanton Solothurn beispielsweise würde eine IKA lediglich 5255.25 CHF kosten, während die gleiche Untersuchung am Inselspital mit 6158 CHF zu Buche schlägt. Warum das SMB gerade die allerteuerste Variante der möglichen Kostenberechnungen wählt, wird nicht begründet. In der Regel muss ein unkompliziertes IKA mit einer Übernachtung sogar mit einem Kostengewicht (cost weight) von nur 0.355 abgerechnet werden. Die tiefste Baserate (unterer Outlier) begründet sich gemäss Recherchen des VEMS Ethical Boards wie folgt: Bei nur einer Übernachtung ist explizit wegen einer 1-Belegungs-DRG immer ein Abschlag in Kauf zu nehmen. Falls der Patient nach Codierung tatsächlich auf F49E codiert wird, bedeutet dies einen Abschlag von 0.184 auf den cost weight von 0.539 . Wir erhalten also ein effektives cost weight von 0.355 , womit sich beispielsweise für eine nicht-universitäre kardiologische Klinik im Kanton Bern Gesamtkosten von 3503.85 CHF ergeben und für eine nicht-universitäre kardiologische Klinik im Kanton Zürich 3372.50 CHF. Daraus hätten sich korrekte Mittelwerte errechnen lassen. Das SMB indes geht bei seinen Kostenvergleichen von komplett falschen Zahlen aus und kommt auf Gesamtkosten von 6158 CHF. Es fällt bei so grundlegenden Rechenfehlern schwer, Erwägungen des SMB bezüglich der Wirtschaftlichkeit der untersuchten Verfahren ernst zu nehmen.

\section{Folgen für den Arzt}

Der Bericht des SMBs fusst also auf mangelhaften Grundlagen, seine Empfehlungen sind in der Lage, einen in der Kardiologie gutetablierten Behandlungspfad umzupflügen - mit gefährlichen Folgen. Aber es handelt sich hier ja nur um eine Empfehlung, könnte man einwenden. Auf Seite 36 seines Berichts hält das SMB nun aber fest: «Aus den obigen Ausführungen folgt, dass in allen Fällen, in denen sich eine IKA durch ein NGCCT-Verfahren substituieren lässt, nur das letztere WZW-konform ist.» (Anm. der Autoren: NGCCT $=$ New Generation Cardiac Computed Tomography). Und weiter: «Das heisst, dass die Krankenkassen in diesen Fällen gemäss Art. 56 Abs. 2 KVG die Vergütung verweigern können.»

Damit wird der Rahmen einer Empfehlung klar überschritten, indem eine Forderung an die Krankenkassen gestellt wird. Diese können nun, gestützt auf den Bericht des SMB, Zahlungen verweigern. Auf einen Bericht notabene, bei dem mehr als fragwürdig ist, ob ihm die Fachgesellschaften, namentlich die beiden vom SMB beigezogenen Fachspezialisten, in dieser Form zustimmen können.

Die vollständige Stellungnahme des VEMS Ethical Boards am Fachbericht des Medical Boards zu Computertomographie-Scannern in der Abklärung der koronaren Herzerkrankung und die dazu gehörenden Referenzen sind im Internet abrufbar unter www.vems.ch/ ethical-board und unter www.physicianprofiling.ch/ VEMSEthicalBoardCT032013.pdf 